\title{
Diagnosis and management of subdural hematoma: a review of recent literature
}

\author{
Wed Alshora $^{1}$, Mohammed Alfageeh ${ }^{2}$, Salman Alshahrani $^{3}$, Saeed Alqahtani, \\ Abdulrahman Dajam ${ }^{3}$, Mutaz Matar ${ }^{4}$, Rakan Ashour ${ }^{5}$, Atif Alasmari ${ }^{3}$, Abdulrhman Alqhtani ${ }^{3}$
}

\author{
${ }^{1}$ Cairo University, Cairo, Egypt \\ ${ }^{2}$ Faculty of Surgery, Umm Al-Qura University, Al Qunfudhah, KSA \\ ${ }^{3}$ King Khalid University, Abha, KSA \\ ${ }^{4}$ Umm Al-Qura University, Mecca, KSA \\ ${ }^{5}$ Al Noor Hospital, Mecca, KSA
}

Received: 22 July 2018

Accepted: 10 August 2018

\section{*Correspondence:}

Dr. Wed Alshora,

E-mail: wedalsh@hotmail.com

Copyright: (C) the author(s), publisher and licensee Medip Academy. This is an open-access article distributed under the terms of the Creative Commons Attribution Non-Commercial License, which permits unrestricted non-commercial use, distribution, and reproduction in any medium, provided the original work is properly cited.

\begin{abstract}
Subdural hematoma is extra-cerebral accumulation of blood between the dura matter and the subarachnoid layer. Subdural hematoma can be associated with significant long-term morbidities and high rates of mortality. The mortality following subdural hematoma can be as high as $32 \%$, and recurrence rates can reach $33 \%$. Acute subdural hematoma is an emergency and requires prompt diagnosis using CT most of the time, and management requires surgery as well as reversal of anticoagulants. We conducted this review using a comprehensive search of MEDLINE, PubMed, and EMBASE, January 1985, through February 2017. The following search terms were used: emergency management of subdural hematoma, subdural hematoma, CT vs. MRI in diagnosis of subdural hematoma, treatment of subdural hematoma. In this review, our aim is to study the etiology of subdural hematoma and understand how it should be diagnosed and managed. Subdural hematoma are clinical emergencies that require immediate and rapid management to prevent significant morbidity and mortality. They can be grouped into acute, subacute, or chronic, with the acute type being the most dangerous and associated with the highest mortality rates. Subdural hematoma is diagnosed using CT or MRI imaging. Management of a patient with subdural hematoma includes resuscitation followed by control of the bleeding. Controlling intracranial pressure is an important factor for predicting the outcomes of subdural hematoma, and should thus be continuously monitored and corrected.
\end{abstract}

Keywords: Subdural hematoma, Diagnosis in emergency room for subdural hematoma, CT vs. MRI, Management of subdural hematoma

\section{INTRODUCTION}

Subdural hematomas are relatively commonly encountered cases that always face neurosurgeons. Incidence and prevalence of subdural hematomas have been increasing lately, with this increase being more prominent in older individuals that could reach 80 cases per 100,000 person-years. ${ }^{1}$ Generally, males have a higher incidence and prevalence of subdural hematomas than females, and it is estimated that about $20 \%$ of subdural hematomas in males are bilateral at presentation. ${ }^{2}$ Although incidence in younger populations is less than older populations, infants and toddlers have a relatively higher incidence when they are compared to other age groups, with an incidence that can reach 40 per 100,000 person-years. Etiologies of these cases in infants and toddlers could be attributed to causes different than other age groups. These etiologies could include 
hydrocephalus, trauma during birth, hematologic diseases, genetic diseases, infections and sepsis, and vascular malformations. $^{3}$

Subdural hematoma can be associated with significant long-term morbidities and high rates of mortality. In fact, it is estimated that mortality following subdural hematoma can be as high as $32 \%$, and recurrence rates can reach $33 \%$. Moreover, in cases of traumatic acute subdural hematoma, mortality can reach $90 \%$ in some clinical settings. Glasgow coma scale has been found to be inversely related with mortality rates. ${ }^{4}$

Subdural hematomas originate from the inner layer of the dura, and out of the arachnoid resulting in multiple septations that contain blood. This nature of subdural hematoma make its management quite challenging and lead to high recurrence rates. ${ }^{5}$ There are many factors that aid the expansion of subdural hematomas. These include the recurrence of bleeding that could be due to elevated levels of fibrinogen and plasmin, the liquefaction of subdural hematomas leading to an osmotic gradient and an enlarging subdural hematoma compartment, and the inflammatory process that is associated with neovascularization leading to more bleeding. 5

Subdural hematomas are categorized into acute, subacute, and chronic. Acute subdural hematoma presents within 3 days of the bleeding, subacute subdural hematoma presents within four to twenty days of the bleeding, and chronic subdural hematoma presents any time after twenty days of the bleeding. The diagnostic methods of choice for the diagnosis of subdural hematomas are CT or MRI imaging techniques. Most acute subdural hematoma cases can be attributed to venous bleeding following traumatic rupture of bridging veins, and leading to increased intracranial pressure. About $20 \%$ of acute subdural hematoma cases can be due to an arterial rupture. ${ }^{6}$

When dealing with patients who suffer from a traumatic brain injury, the prevalence of subdural hematomas can reach $20 \%$, with about half of these patients presenting in a coma. ${ }^{7}$ On the other hand, the use of antiplatelet and anticoagulation drugs can also lead to the formation of a subdural hematoma. ${ }^{7}$ However, infantile subdural hematoma has not been well understood yet.

\section{METHODS}

We did a systematic search for subdural hematoma using PubMed search engine (http://www.ncbi.nlm.nih.gov/) and Google Scholar search engine (https://scholar. google.com). Our search also looked for the prevalence, etiologies, diagnosis, and treatment of subdural hematoma cases. All relevant studies were retrieved and discussed. We only included full articles. The following search terms were used: emergency management of subdural hematoma, subdural hematoma, CT vs. MRI in diagnosis of subdural hematoma, treatment of subdural hematoma.
The study was approved by the review board of King AbdulAziz Hospital.

\section{Initial management of SDH patients}

When dealing with either acute, subacute, or chronic subdural hematoma, management must start with applying advanced trauma life support protocols. In the recent guidelines, the Neuro-Critical Care Society recommend maintaining a mean arterial pressure between 80 and $110 \mathrm{mmHg}$, with a systolic arterial pressure that does not exceed $180 \mathrm{mmHg}$. These targets aim at the maintenance of a sufficient cerebral perfusion with the prevention of continuous hemorrhage. ${ }^{8}$ Oxygenation of the brain must be kept at $60 \mathrm{mmHg}$, as hypoxia can lead to significant permanent damage. ${ }^{9}$ In some cases of severe injuries, agitation, intoxication, or altered mental status, intubation is administrated to achieve sufficient oxygenation and to decrease aspiration risk. ${ }^{10}$ To provide sufficient sedation in trauma settings, etomidate is usually used for induction along with propofol, ketamine, and fentanyl. Intravenous lidocaine is also usually administrated to prevent elevated intracranial pressure. ${ }^{9}$ To achieve sufficient neuromuscular blockade, intravenous succinylcholine, rocuronium, or cisatracurium could be used. ${ }^{9}$ After intubation is achieved, sedatives that are used to maintain the sedation status include propofol, midazolam, fentanyl, dexmedetomidine, or remifentanil.

\section{Imaging to confirm the diagnosis}

After achieving sufficient resuscitation of the patient, intracranial imaging is required to confirm the diagnosis of subdural hematoma and plan proper management. The use of CT scan imaging in the detection of subdural hematoma has several advantages including the wide availability, rapid results, and high sensitivity and specificity, which can reach $96 \%$ and $98 \%$ respectively. On the other hand, MRI imaging techniques are still preferred due to sensitivity and specificity that reach $100 \%$, the ability to detect even minimal bleeding, and the ability to identify etiologies.

Both CT and MRI imaging techniques can give information on the size of the hematoma, chronicity of the bleeding, medline shifting (if present), effacement of basal cistern, and trapping of the ventricles. When the etiology is suspected to be of vascular origins, patients are recommended to further undergo MRA. Emergent imaging is indicated in any case that shows dramatic deterioration of clinical or mental status. ${ }^{6}$

\section{Management of intracranial hypertension in subdural hematoma}

Usually, subdural hematoma is associated with a significant increase in intracranial pressure. Unfortunately, an intracranial pressure that is higher than 22 has been associated with worse survival outcomes, and higher morbidity and mortality in patients who 
present with subdural hemorrhage. ${ }^{11}$ Therefore, management of increased intracranial pressure is of extreme importance in subdural hematomas. Initially, simple measures like head elevation could be applied. These will help in preventing obstruction of venous outflow. ${ }^{12}$ Another important measure is hyperventilation that will lead to vasoconstriction and a decrease in intracranial pressure. ${ }^{11}$ However, long-term hyperventilation has been associated with ischemia and alkalosis, therefore it should be cautiously used. In fact, a clinical trial has shown that applying hyperventilation in patients with traumatic brain injury for five days was associated with worse outcomes. ${ }^{13}$ Hypertonic saline and hyperosmotic saline could also be beneficial as they promote the shift of fluids into the circulation and out of cerebral intracellular compartments. However, there use should be temporary. The placement of a shunt that will drain CSF is not recommended due to the risk of stretching veins. Several factors can aid in determining the use of hyperosmolar saline versus hypertonic saline. These include volume status, history and comorbidities. For example, if the patient is hypotensive, hypertonic therapy is preferred. On the other hand, patients who have a history of congestive heart failure are recommended to take mannitol. High sodium levels (more than $165 \mathrm{mEq} / \mathrm{L}$ ) have also been associated with poor prognosis. However, correcting sodium levels has not been found to improve outcomes, or decrease intracranial pressure. ${ }^{14}$ Hypothermia could also have an effect in lowering intracranial pressure and improving outcomes of subdural hematoma. However, this effects is still studied in clinical trials with no clear evidence to support this. ${ }^{14}$ Moreover, hypothermia could potentially lead to other complications like arrhythmias. ${ }^{14}$

\section{Antiplatelet and anticoagulation agents and subdural hematoma}

The use of antiplatelet and anticoagulation agents is widely prevalent which led to significant increase in the incidence of spontaneous subdural hematomas. These cases of subdural hematoma can sometimes be difficult to reverse. ${ }^{15}$ To achieve better outcomes in these cases, rapid reversal of anticoagulation is essential. ${ }^{16}$ Patients who are on warfarin and who develop a spontaneous subdural hemorrhage should immediately receive vitamin $\mathrm{K}$ and prothrombin complex concentrate, with INR being continuously monitored. In severe emergency cases, recombinant factor VIIa could also be used. ${ }^{17}$

When dealing with spontaneous subdural hematoma in patients who are on dabigatran, apixaban, rivaroxaban, or edoxaban, the use of the monoclonal antibody idarucizumab is recommended. Other options in this population include plasmapheresis and hemodialysis. ${ }^{16}$ The use of platelet transfusions in this specific population has been found to cause higher mortality rates. ${ }^{16}$

The presence of coagulopathy is not limited to spontaneous subdural hematoma. About a third of patients who have subdural hematoma due to a traumatic brain injury can have severe coagulopathy. This can even lead to the development of disseminated intravascular coagulation (DIC). Management of disseminated intravascular coagulation include coagulation factors, cryoprecipitate, and prothrombin complex concentrate transfusion. $^{18}$

The return to anticoagulation agents use is still a controversial issue. The American College of Chest Physicians recommend the discussion of anticoagulants resumption on a case basis, with taking into consideration the severity of hematoma, risk of subdural hematoma recurrence, and risk of thrombosis. ${ }^{19}$

\section{Management of seizures in subdural hematoma}

Prophylaxis against seizures is still an area of debate in subdural hematoma cases. Previous reports have suggested that up to $25 \%$ of patients with traumatic subdural hematoma will develop seizures at least once through their clinical course. ${ }^{20}$ Recent guidelines have recommended the use of phenytoin as a prophylaxis of seizures in patients who present with a traumatic subdural hematoma. $^{21}$ Seizures can be associated with poor outcomes due to their effects in increasing metabolism and elevating intracranial pressure. ${ }^{20}$ More recently, the use of levetiracetam in seizures prophylaxis for subdural hematoma patients has been increasing. ${ }^{22}$

However, when dealing with elderly patients with chronic subdural hematoma, no evidence is present to support the use of anti-seizures prophylaxis. Won et al suggested the use of a scoring system in this population to assess their risk of seizures and thus their need of prophylactic therapy. This score takes several factors into consideration including the use of anticoagulation drugs, the need for surgery, the presence of seizures history, along with other factors. ${ }^{23}$

In conclusion, continuous research on anti-seizures drugs and their use in subdural hematoma is still required to establish their use for different presentations and scenarios.

\section{Indication for surgical interventions}

As mentioned above, asymptomatic and mild cases of subdural hematoma can be treated medically. However, in moderate to severe cases, or when clinical deterioration is present, surgical intervention is indicated. Recurrent subdural hematomas can also be an indication for surgical intervention. A recurrent subdural hematoma is defined as the re-accumulation of the bleeding that becomes symptomatic again. Altered mental status, dilatation of both pupils, or extensor posturing are also alarming signs that require immediate surgical intervention. As a general rule, subdural hematomas that are larger than $10 \mathrm{~mm}$ can be considered for surgical intervention. There are several surgical techniques that 
are used in cases of subdural hematoma. These include twist drill craniostomy, burr hole(s), craniotomy, along with other procedures that we will discuss. ${ }^{4}$

\section{Twist drill craniostomy procedure for the treatment of subdural hematoma}

The twist drill craniostomy procedure for the treatment of subdural hematoma was first introduced in $1966 .{ }^{24}$ Over time, the procedure have improved significantly with the introduction of newer more advanced techniques, and with more profound studying of ideal drilling angles, points to enter, drilling methods, use of screws, and irrigation ports. Moreover, the twist drill craniostomy procedure has been associated with relatively low rate of complications when compared to other surgical interventions. Despite being low, these complications can include seizures, infections, and subdural hematoma recurrence. Severs complications like pneumocephalus are very rare. ${ }^{25}$

The SEPS technique was later described in 1999 and led to significantly better outcomes with even lower complications and recurrence rates. In this procedure, a port with threads are entered the thick portion of the subdural hematoma without going into the subdural space itself. Application of this allows drainage slowly with the concepts of negative pressure. ${ }^{26}$ It was estimated that SEPS procedure has a success rate that can reach $77 \%$. However, this success rate is lower in patients who have septated or mixed density subdural hematomas. ${ }^{27}$ In SEPS technique, it was found that head elevation degree is not associated with changes in drainage or recurrence risk. Some studies have suggested that following SEPS, up to $20 \%$ may need to further undergo burr holes procedure. $^{28}$

When performing twist drill craniostomy on elderly patients with subdural hematoma, local anesthesia could offer a better option, especially in patients who have other comorbidities. To avoid the occurrence of damage to the cerebral parenchyma, a previous study has suggested the use an infant feeding tube while draining the hemorrhage. ${ }^{29}$ Generally, the twist drill craniostomy procedure has been found in several studies to be associated with faster recovery, shorter hospital stays, and less complications when compared with burr holes. ${ }^{30}$

Moreover, sites of entry and duration of drainage when performing a twist drill craniostomy can play a significant role in the determination of outcomes. When entry is done on the thick portions of the subdural hematoma, efficacy of twist drill craniostomy has been found to increase in efficacy. Other key factors include the use of ports that will allow the injection of other therapies directly into the cavity of the subdural hematoma. A more recent model of twist drill craniostomy uses two ports for both drainage and irrigation. $^{31}$
The use of tPA during and after twist drill craniostomy has also been associated with better outcomes. A previous trial has found that recurrence rates can be lowered to zero when using tPA. The use of urokinase has also been associated with better outcomes, shorter hospital stays, and recurrence rates that are as low as $0.4 \% .^{32}$

\section{Burr hole evacuation for the treatment of subdural hematoma}

The burr hole evacuation technique is considered to be one of the most common and widely used procedures in the surgical treatment of subdural hematoma. To perform a burr hole evacuation, an environment that achieves all conditions of surgical operation is required. Usually, the burr hole measures about $14 \mathrm{~mm}$ allowing for a good visualization of the hematoma, the detection of clots (if present), faster access and control of the hemorrhage, and the ability to use other additional surgical instruments like endoscopes, which are not achievable in twist drill craniostomy. ${ }^{33}$

Burr hole evacuation is available both under local or general anesthesia. It has been estimated that burr hole evacuation under local anesthesia could achieve $98.6 \%$ evacuation with a recurrent rate that is $4.7 \% .^{33}$ The use of single versus multiple holes when performing a burr hole evacuation procedure has also been studied. It was reported that multiple burr holes were associated with significantly less recurrent rates. ${ }^{34}$

The most important factor to determine success rates following a burr hole procedure is the replacement of drain. Drain replacement, actually, was found to lead to significantly lower recurrence rates when compared to burr hole evacuation procedure without drain. ${ }^{35}$ Complications and mortality rates following the procedure were not found to significantly differ with or without drain replacement. ${ }^{36}$ Moreover, the placement of a drain even in a single burr hole procedure was associated with improved outcomes and lower recurrence rates when compared to multiple burr holes procedure. ${ }^{37}$

The time for which draining continues also seems to have an effect. Previous studies have found that continuing drainage for a period longer than three days was associated with significant decrease in recurrence rates. The site of drain replacement could also play a role in determining outcomes, reducing the risk of seizures, and leading to less infections. Moreover, the use of subperiosteal drain was associated with significantly lower rates of recurrence when compared to subdural drains. ${ }^{38}$

\section{Craniotomy in the management of acute subdural hematoma}

Patients who present with an acute subdural hematoma accompanied by thick clots are recommended to undergo 
craniotomy. Other cases in which craniotomy is recommended include chronic or subacute subdural hematoma where other surgical procedures have not been successful. Generally, acute subdural hematoma is considered a life threatening condition that is associated with significantly high mortality rates. Therefore, surgical intervention with craniotomy is indicated immediately in these cases. $^{39}$

Outcomes of craniotomy for acute subdural hematoma can be affected by multiple factors including the cause of the hematoma, age of the patient, the time before surgery, intracranial pressure status, and the size of the hematoma. Despite all measure, subdural hematoma remains to have a relatively high mortality rates. Possible reasons behind this high mortality include injury to the cerebral parenchyma, autoregulation dysfunctions, altered mental and neurologic states, and high intracranial pressure in most cases. ${ }^{7}$ In fact, it is estimated that patients with acute subdural hematoma who present with a Glasgow coma score of three of four, have a mortality rate that is as high as $90 \%$. $^{40}$

\section{CONCLUSION}

Subdural hematomas are clinical emergencies that require immediate and rapid management to prevent significant morbidity and mortality. Subdural hematoma can be grouped into acute, subacute, or chronic, with the acute type being the most dangerous and associated with the highest mortality rates. Subdural hematoma is mainly diagnosed using CT or MRI imaging. The first step in the management of a patient with subdural hematoma is appropriate resuscitation. Later, further methods to control the bleeding could be applied. Intracranial pressure is an important factor controlling outcomes of subdural hematoma and should thus be continuously monitored and corrected. Although most cases of subdural hematoma are traumatic, some other cases are spontaneous, and are in most cases a result of underlying coagulopathy. In these cases, the etiology should be determined and treated accordingly to stop the hemorrhage and prevent further accumulation of blood. In moderate to severe cases, where medical and conservative management is not enough, surgical intervention is indicated to improve survival and prevent possible complications and mortality.

Funding: No funding sources Conflict of interest: None declared

Ethical approval: Not required

\section{REFERENCES}

1. Balser D, Farooq S, Mehmood T, Reyes M, Samadani U. Actual and projected incidence rates for chronic subdural hematomas in United States veterans administration and civilian populations. J Neurosurg. 2015;123(5):1209-15.
2. Kudo H, Kuwamura K, Izawa I, Sawa H, Tamaki N. Chronic subdural hematoma in elderly people: present status on Awaji Island and epidemiological prospect. Neurol Med Chir (Tokyo). 1992;32(4):207-9.

3. Talvik I, Metsvaht T, Leito K, Põder H, Kool P, Väli M, et al. Inflicted traumatic brain injury (ITBI) or shaken baby syndrome (SBS) in Estonia. Acta Paediatr. 2006;95(7):799-804.

4. Tanikawa M, Mase M, Yamada K, Yamashita N, Matsumoto T, Banno T, et al. Surgical treatment of chronic subdural hematoma based on intrahematomal membrane structure on MRI. Acta Neurochir. 2001;143(6):613-8.

5. Lee JY, Ebel H, Ernestus RI, Klug N. Various surgical treatments of chronic subdural hematoma and outcome in 172 patients: is membranectomy necessary? Surg Neurol. 2004;61(6):523-7.

6. Maxeiner H, Wolff M. Pure subdural hematomas: a postmortem analysis of their form and bleeding points. Neurosurgery. 2002;50(3):503-8.

7. Bullock MR, Chesnut R, Ghajar J, Gordon D, Hartl $\mathrm{R}$, Newell DW, et al. Surgical management of acute subdural hematomas. Surgical Management of Traumatic Brain Injury Author Group. Neurosurgery. 2006;58(3):16-24.

8. Smith MD, Kishikova L, Norris JM. Surgical management of chronic subdural haematoma: one hole or two? Int J Surg. 2012;10(9):450-2.

9. Rajajee V, Riggs B, Seder DB. Emergency neurological life support: airway, ventilation, and sedation. Neurocrit Care. 2017;27(1):4-28.

10. Bernard SA, Nguyen V, Cameron P, Masci K, Fitzgerald M, Cooper DJ, et al. Prehospital rapid sequence intubation improves functional outcome for patients with severe traumatic brain injury: a randomized controlled trial. Ann Surg. 2010;252(6):959-65.

11. Badri S, Chen J, Barber J, Temkin NR, Dikmen SS, Chesnut RM. Mortality and long-term functional outcome associated with intracranial pressure after traumatic brain injury. Intensive Care Med. 2012;38(11):1800-9.

12. Mayer SA, Chong JY. Critical care management of increased intracranial pressure. J Int Care Med. 2002;17(2):55-67.

13. Muizelaar JP,Marmarou A, Ward JD, Kontos HA, Choi SC, Becker DP, et al. Adverse effects of prolonged hyperventilation in patients with severe head injury: a randomized clinical trial. J Neurosurg. 1991;75(5):731-9.

14. Andrews PJ, Sinclair HL, Rodriguez A, Harris BA, Battison CG, Rhodes JK, et al. Eurotherm3235 trial collaborators. Hypothermia for intracranial hypertension after traumatic brain injury. $\mathrm{N}$ Engl J Med. 2015;373(25):2403-12.

15. Gaist D, García Rodríguez LA, Hellfritzsch M, Poulsen FR, Halle B, Hallas J, et al. Association of antithrombotic drug use with subdural hematoma risk. JAMA. 2017;317(8):836-46. 
16. Frontera JA, Lewin JJ 3rd, Rabinstein AA, Aisiku IP, Alexandrov AW, Cook AM, et al. Guideline for reversal of antithrombotics in intracranial hemorrhage: A Statement for Healthcare Professionals from the Neurocritical Care Society and Society of Critical Care Medicine. Neurocrit Care. 2016;24(1):6-46.

17. Desborough MJ, Oakland KA, Landoni G, CrivellariM, Doree C, Estcourt LJ, et al. Desmopressin for treatment of platelet dysfunction and reversal of antiplatelet agents: a systematic review and meta-analysis of randomized controlled trials. J Thromb Haemost. 2017;15(2):263-72.

18. Allard CB, Scarpelini S, Rhind SG, Baker AJ, Shek $\mathrm{PN}$, Tien $\mathrm{H}$, et al. Abnormal coagulation tests are associated with progression of traumatic intracranial hemorrhage. J Trauma. 2009;67(5):959-67.

19. Hirsh J, Guyatt G, Albers GW, Harrington R, Schünemann HJ. Executive summary: American College of Chest Physicians Evidence-Based Clinical Practice Guidelines (8th Edition). Chest. 2008;133(6):71-109.

20. Rabinstein AA, Chung SY, Rudzinski LA, Lanzino G. Seizures after evacuation of subdural hematomas: incidence, risk factors, and functional impact. J Neurosurg. 2010;112(2):455-60.

21. Carney N, Totten AM, O'Reilly C, Ullman JS, Hawryluk GW, Bell MJ, et al. Guidelines for the management of severe traumatic brain injury, Fourth Edition. Neurosurgery. 2017;80(1):6-15.

22. Inaba K, Menaker J, Branco BC, Gooch J, Okoye OT, Herrold J, et al. A prospective multicenter comparison of levetiracetam versus phenytoin for early posttraumatic seizure prophylaxis. J Trauma Acute Care Surg. 2013;74(3):766-71.

23. Won SY, Dubinski D, Bruder M, Cattani A, Seifert V, Konczalla J. Acute subdural hematoma in patients on oral anticoagulant therapy:management and outcome. Neurosurg Focus. 2017;43(5):12.

24. Rand BO, Ward AA Jr, White LE Jr. The use of the twist drill to evaluate head trauma. J Neurosurg. 1966;25(4):410-5.

25. Caron JL, Worthington C, Bertrand G. Tension pneumocephalus after evacuation of chronic subdural hematoma and subsequent treatment with continuous lumbar subarachnoid infusion and craniostomy drainage. Neurosurgery. 1985;16(1):107-10.

26. Asfora WT, Schwebach L. Amodified technique to treat chronic and subacute subdural hematoma: technical note. Surg Neurol. 2003;59(4):329-32.

27. Neal MT, Hsu W, Urban JE, Angelo NM, Sweasey TA, Branch CL Jr. The subdural evacuation port system: outcomes from a single institution experience and predictors of success. Clin Neurol Neurosurg. 2013;115(6):658-64.

28. Krieg SM, Aldinger F, Stoffel M, Meyer B, Kreutzer J. Minimally invasive decompression of chronic subdural haematomas using hollow screws: efficacy and safety in a consecutive series of 320 cases. Acta Neurochir. 2012;154(4):699-705.

29. Yadav YR, Yadav S, Parihar VS. Modified twist drill technique in the management of chronic subdural hematoma. Turk Neurosurg. 2013;23(1):50-4.

30. Balser D, Rodgers SD, Johnson B, Shi C, Tabak E, Samadani U. Evolving management of symptomatic chronic subdural hematoma: experience of a single institution and review of the literature. Neurol Res. 2013;35(3):233-42.

31. Wang QF, Cheng C, You C. A new modified twist drill craniostomy using a novel device to evacuate chronic subdural hematoma. Medicine (Baltimore). 2016;95(10):e3036.

32. Lu J, Shen D, Hu F, Zhou J, Lan F, Guo D, et al. An improved electronic twist-drill craniostomy procedure with post-operative urokinase instillation in treating chronic subdural hematoma. Clin Neurol Neurosurg. 2015;136:61-5.

33. Khadka NK, Sharma GR, Roka YB, Kumar P, Bista $\mathrm{P}$, Adhikari D, et al. Single burr hole drainage for chronic subdural haematoma. Nepal Med Coll J. 2008;10(4):254-7.

34. Taussky P, Fandino J, Landolt H. Number of burr holes as independent predictor of postoperative recurrence in chronic subdural haematoma. $\mathrm{Br} \mathrm{J}$ Neurosurg. 2008;22(2):279-82.

35. Gazzeri R, GalarzaM, NeroniM, Canova A, Refice GM, Esposito S. Continuous subgaleal suction drainage for the treatment of chronic subdural haematoma. Acta Neurochir. 2007;149(5):487-93.

36. Singh AK, Suryanarayanan B, Choudhary A, Prasad A, Singh S, Gupta LN. A prospective randomized study of use of drain versus no drain after burr-hole evacuation of chronic subdural hematoma. Neurol India. 2014;62(2):169-74.

37. Kutty SA, Johny M. Chronic subdural hematoma: a comparison of recurrence rates following burr-hole craniostomy with and without drains. Turk Neurosurg. 2014;24(4):494-7.

38. Zumofen D, Regli L, Levivier M, Krayenbühl N. Chronic subdural hematomas treated by burr hole trepanation and a subperiostal drainage system. Neurosurgery. 2009;64(6):1116-21.

39. Servadei F, NasiMT, Cremonini AM, Giuliani G, Cenni P, Nanni A. Importance of a reliable admission Glasgow Coma Scale score for determining the need for evacuation of posttraumatic subdural hematomas: a prospective study of 65 patients. J Trauma. 1998;44(5):868-73.

40. Koç RK, Akdemir H, Oktem IS, Meral M, Menkü A. Acute subdural hematoma: outcome and outcome prediction. Neurosurg Rev. 1997;20(4):239-44.

Cite this article as: Alshora W, Alfageeh M, Alshahrani S, Alqahtani S, Dajam A, Matar M, et al. Diagnosis and management of subdural hematoma: a review of recent literature. Int J Community Med Public Health 2018;5:3709-14. 